\title{
Developmental Synaptic Depression Underlying Reorganization of Visceral Reflex Pathways in the Spinal Cord
}

\author{
Isao Araki and William C. de Groat \\ Department of Pharmacology, University of Pittsburgh School of Medicine, Pittsburgh, Pennsylvania 15261
}

During development, neuronal connectivity has a remarkable plasticity. Synaptic refinement in the spinal autonomic nucleus might be involved in the elimination of primitive segmental reflexes and the emergence of mature spinobulbospinal reflexes, which occurs a few weeks after birth. To address this possibility, we examined the postnatal changes of segmental excitatory synaptic transmission by applying the whole-cell recording technique to parasympathetic preganglionic neurons in slice preparations of the rat lumbosacral spinal cord. The mean magnitude of unitary excitatory synaptic currents evoked in preganglionic neurons by stimulation of single interneurons remained unchanged during the first two postnatal weeks but was reduced by $50 \%$ during the third postnatal week. This reduction in synaptic efficacy was associated with a decrease in the amount of transmitter release from interneurons. Moreover, this developmental depression of segmental synaptic transmission was prevented by spinal cord transection at the thoracic level on postnatal day 14. Thus, developmental modification of excitatory synapses on preganglionic neurons appears to be attributable to competition between segmental interneuronal and descending bulbospinal inputs, which results in the developmental reorganization of parasympathetic excretory reflex pathways.

Key words: synaptic plasticity; developmental synaptic depression; glutamatergic excitatory synaptic currents; spinal autonomic nucleus; parasympathetic preganglionic neurons; micturition reflex; chronic spinal transection; quantal analysis
During early postnatal life, synaptic refinements are well known to occur in the central (Hubel and Wiesel, 1965; Mendell, 1984; Fregnac et al., 1988; Reiter and Stryker, 1988; Constantine-Paton et al., 1990) and peripheral (Redfern, 1970; Ridge and Betz, 1984; Dan and Poo, 1992; Balice-Gorden and Lichtman, 1994; Colman et al., 1997) nervous systems. This synaptic remodeling serves to refine initial coarse-grained and exuberant neuronal connections and results in formation of highly tuned neural circuits (Purves and Lichtman, 1985; Goodman and Shatz, 1993). In spinal autonomic reflex pathways, synaptic refinement could be involved in the elimination of primitive segmental reflexes and the emergence of mature reflex patterns. In neonates of many species, excretory functions (micturition and defecation) are mediated exclusively by the segmental parasympathetic reflex pathway, which is activated when the mother licks the perineum of the neonate (Beach, 1966; de Groat et al., 1975; Thor et al., 1989). A few weeks after birth, this segmental reflex begins to disappear and is replaced by the newly developed spinobulbospinal reflex as the principal mechanism for excretion (de Groat, 1975; Fukuda et al., 1981; Kruse and de Groat, 1990; de Groat et al., 1993). This is also a critical period for the establishment of synaptic refinements (Purves and Lichtman, 1985) as well as for the functional maturation of descending pathways to the spinal cord (Gilbert and Stelzner, 1979; Mendell, 1984). Because the neuronal connections at developing synapses appear to be refined by competition between multiple synaptic inputs converging on the same target cell (Purves and Lichtman, 1985; Goodman and Shatz,

\footnotetext{
Received May 5, 1997; revised Aug. 18, 1997; accepted Aug. 20, 1997.

This work was supported by National Institute of Diabetes and Digestive and Kidney Diseases Grants DK 37241 and DK 49430. We thank Dr. Motoy Kuno for his critical comments.

Correspondence should be addressed to Isao Araki, Clinical Research Center and Department of Urology, Utano National Hospital, Narutaki, Kyoto 616, Japan. Copyright (C) 1997 Society for Neuroscience $0270-6474 / 97 / 178402-06 \$ 05.00 / 0$
}

1993), it is possible that the segmental autonomic pathway operating in the early postnatal life is suppressed by competition with the descending projection from the brain for synaptic connections to parasympathetic preganglionic neurons (PGNs). This view is supported by the finding that the segmental parasympathetic reflex can be restored in adult animals when the descending tract is impaired by chronic spinal cord transection (de Groat et al., 1975, 1993; Kruse and de Groat, 1990).

To address this possibility, we examined the postnatal changes in synaptic transmission between segmental interneurons and PGNs in the lumbosacral parasympathetic nucleus (SPN) of neonatal rats (Fig. 1). Interneurons located just dorsal to the SPN

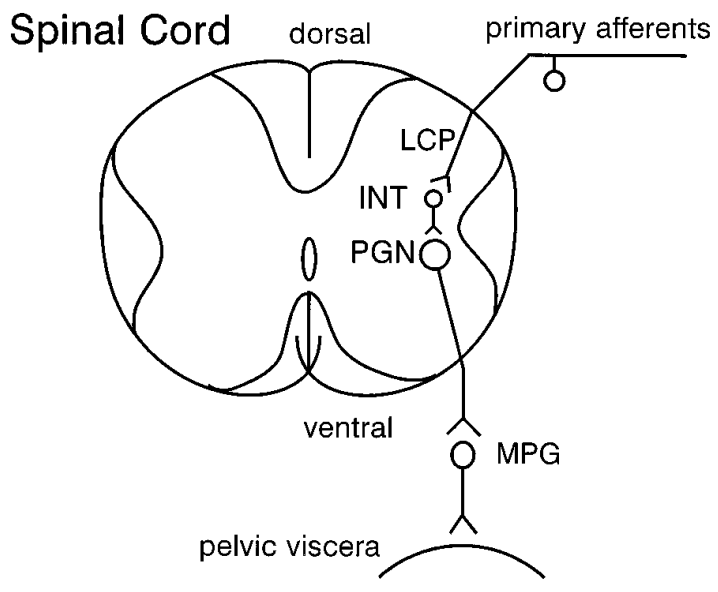

Figure 1. Schematic diagram for the neural circuit of parasympathetic spinal segmental reflex and the neurons studied in slice preparations of the lumbosacral cord. $P G N$, Parasympathetic preganglionic neurons; INT, dorsal interneurons; $L C P$, lateral collateral pathway of primary afferents; $M P G$, major pelvic ganglia. 
A

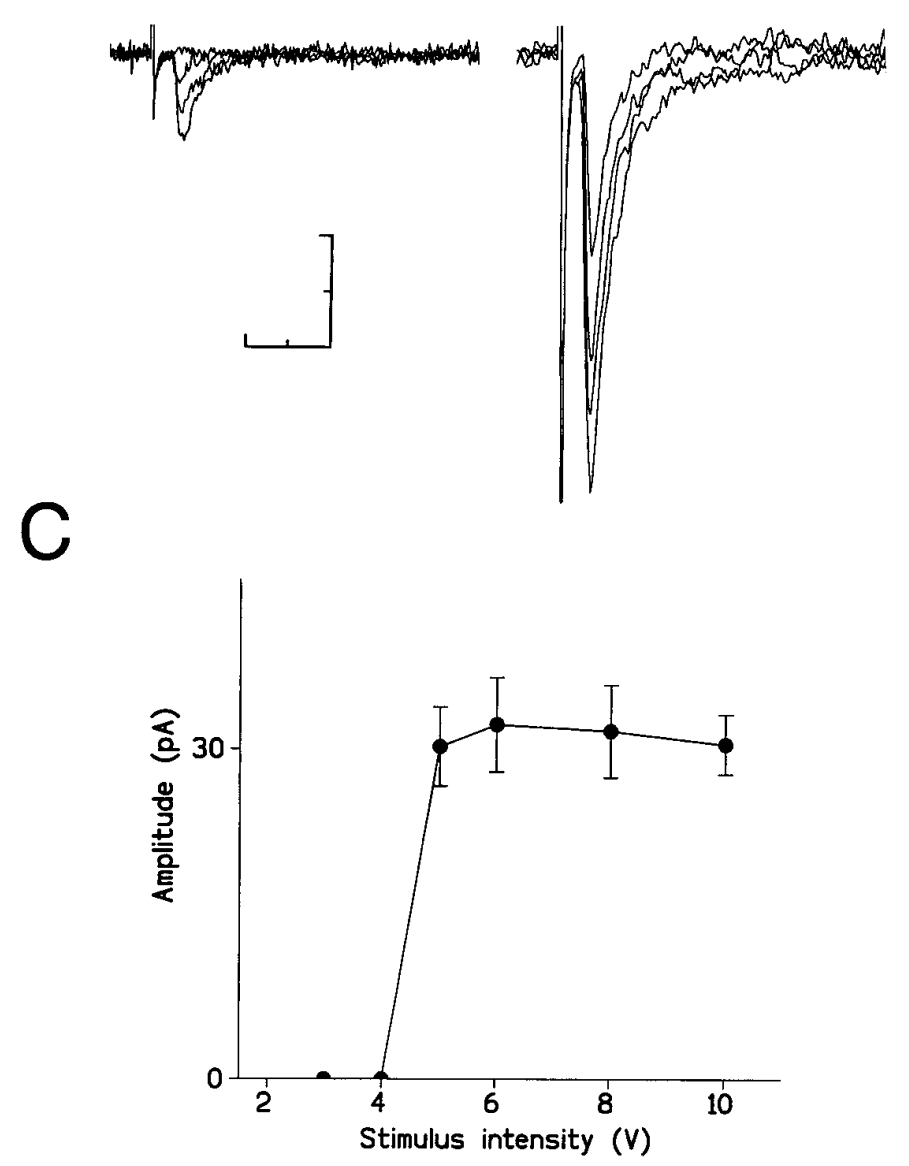

B
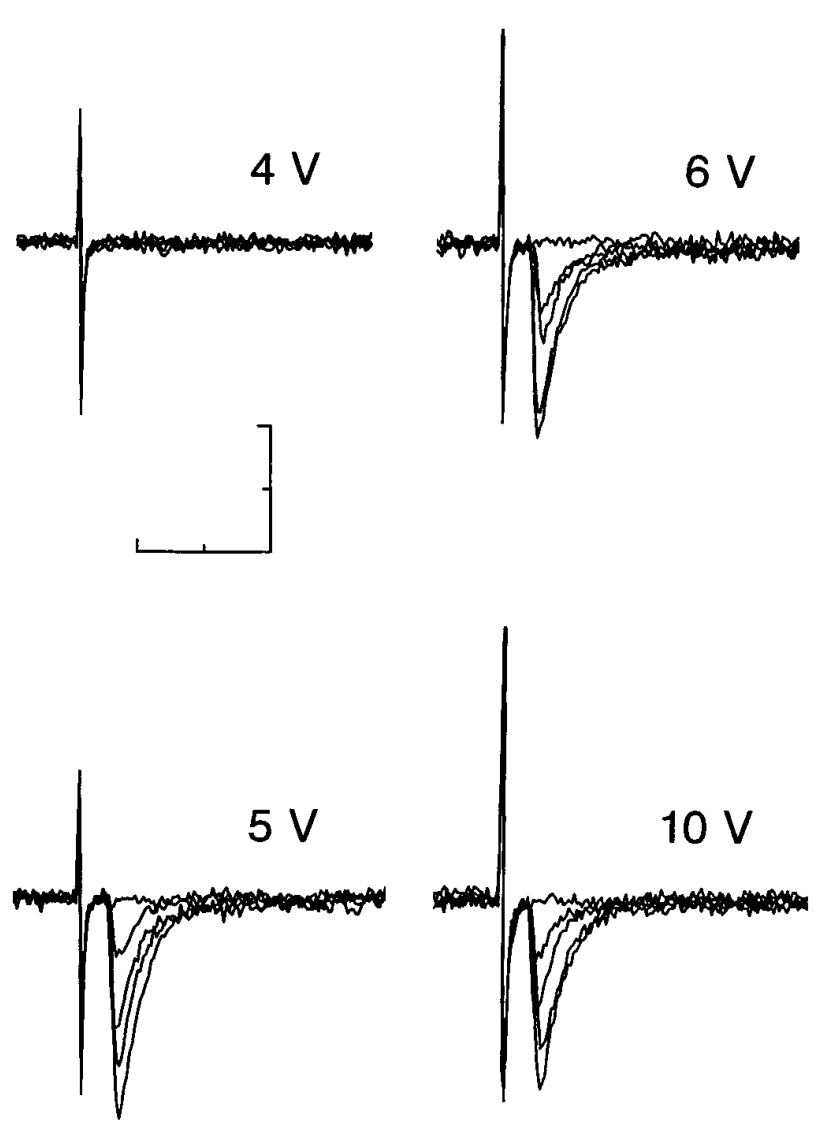

Figure 2. EPSCs in segmental parasympathetic reflex pathways. $A$, Composite EPSCs evoked in a dorsal interneuron by stimulation of primary afferent fibers. Four responses evoked by threshold (left) or supramaximal (right) intensities of stimulus are superimposed. B, Unitary EPSCs evoked in a PGN by stimulation of a dorsal interneuron. Five responses at each stimulus intensity indicated above tracing are superimposed. $C$, Relationship between the stimulus intensity and the mean peak amplitude of 30 consecutive EPSCs (including failures) in the same cell as in B. Vertical bars represent SEM. The holding potential was $-60 \mathrm{mV}$. Scale bars, $10 \mathrm{msec}$ and $40 \mathrm{pA}$.

appear to mediate disynaptic activation of PGNs by primary afferents in segmental parasympathetic reflex pathways, as suggested by previous reports (McMahon and Morrison, 1982; Birder and de Groat, 1992; Nadelhaft and Vera, 1995; Araki and de Groat, 1996; de Groat et al., 1996). The present study shows that the amount of transmitter released from single interneurons to PGNs is abruptly reduced during the third postnatal week. Moreover, this developmental synaptic depression was prevented when the bulbospinal pathways were eliminated by chronic spinal cord transection at the thoracic level. These results suggest that the developmental modification of excitatory synapses on PGNs is attributable to competition between their segmental and suprasegmental synaptic inputs, which results in the reorganization of parasympathetic excretory reflex pathways.

\section{MATERIALS AND METHODS}

Slice preparation. PGNs in the lumbosacral cord were identified by retrograde labeling with a fluorescent dye. For this purpose, $5 \mu \mathrm{l}$ of $4 \%$ fast blue (Polyloy, GrossUmstadt, Germany) was injected into the intraperitoneal space 3-7 d before the electrophysiological studies. PGNs were clearly labeled by this procedure, as reported previously (Anderson and Edwards, 1994; Araki and de Groat, 1996).
Sprague Dawley rats, 6-22 d old, were decapitated under ether anesthesia. The lumbosacral cord (L6-S1) was quickly isolated and sectioned into $120-150 \mu \mathrm{m}$ transverse slices with a vibrating slicer (Vibratome; Technical Products International, St. Louis, MO) as described previously (Edwards et al., 1989). The slices were incubated at $37^{\circ} \mathrm{C}$ for $1 \mathrm{hr}$ and maintained thereafter by continuous perfusion at room temperature.

Patch-clamp recording. The basic procedures for recording whole-cell currents from individual neurons in slice preparations were identical to those reported previously (Manabe et al., 1991; Araki, 1994; Araki and de Groat, 1996). A slice preparation was placed in a recording chamber with a volume of $0.6 \mathrm{ml}$ and continuously superfused by a solution equilibrated with $100 \% \mathrm{O}_{2}$ at a rate of $2.5 \mathrm{ml} / \mathrm{min}$. The bathing solution had the following composition (in $\mathrm{mM}$ ): $130 \mathrm{NaCl}, 5 \mathrm{KCl}, 2 \mathrm{CaCl}_{2}, 1$ $\mathrm{MgCl}_{2}, 11$ glucose, and 10 HEPES, pH 7.4, with NaOH. One micromolar strychnine (Sigma, St. Louis, MO) and $10 \mu \mathrm{M}$ bicuculline methiodide (Sigma) were always present in the bathing solution to eliminate inhibitory synaptic inputs. Each slice of the lumbosacral cord (L6-S1) was surveyed for fast blue-containing neurons (i.e., PGNs) along the intermediolateral border of the gray matter under an upright microscope equipped with fluorescent optics (BH2; Olympus, Tokyo, Japan) (Fig. 1). After identification of a PGN, the neuron was viewed with Nomarski optics, and a patch pipette (3-4 M $\Omega$ ) was applied to record whole-cell currents with the use of an Axopatch 200A amplifier (Axon Instruments, Foster City, CA). The pipette solution contained (in mM): 140 potassium gluconate, $4 \mathrm{NaOH}, 3 \mathrm{MgCl}_{2}, 10 \mathrm{HEPES}$, and 0.2 EGTA, pH 7.3, with 


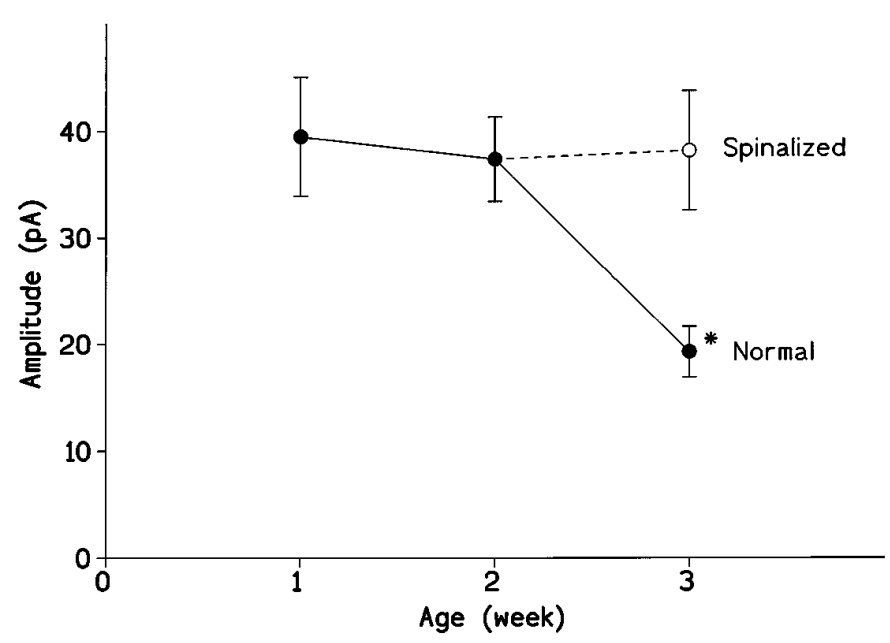

Figure 3. Developmental change in the efficacy of excitatory transmission at interneuronal-PGN synapses and its modification by chronic spinal transection. Mean peak amplitudes of unitary EPSCs recorded from PGNs in intact $(\bullet)$ or spinalized $(\bigcirc)$ rats are plotted against ages. Mean peak amplitudes were measured from averaged responses of $>30$ consecutive EPSCs (including failures). The holding potential was -60 $\mathrm{mV}$. Each point represents the mean \pm SEM (vertical bar) from 14 or 15 cells. The asterisk represents significant difference from the values observed in other preparations $(p<0.006)$.

$\mathrm{KOH}$. The recordings were made at room temperature $\left(22-25^{\circ} \mathrm{C}\right)$. Synaptic responses were evoked in PGNs by electrical stimulation (50-100 $\mu \mathrm{sec})$ with a glass micropipette $(\sim 2 \mathrm{M} \Omega)$ filled with external solution (Fig. 1). The stimulating pipette was placed on the cell body of a dorsal interneuron. The dorsal interneuron was defined as an unlabeled small neuron immediately dorsal to SPN. Stimulus frequency was $1 \mathrm{~Hz}$. To avoid failures of stimulation, stimulus intensities 1.2-1.5 times higher than the threshold were used. When EPSCs were recorded from dorsal interneurons, primary afferent fibers on the lateral edge of the dorsal horn [the lateral collateral pathway (LCP)] (Nadelhaft and Booth, 1984; McKenna and Nadelhaft; 1986) visible with Nomarski optics were stimulated by a glass pipette with a larger tip $(\sim 10 \mu \mathrm{m})$. Cells with high series resistance $(>15 \mathrm{M} \Omega)$ or low input resistance $(<150 \mathrm{M} \Omega)$ were not studied. Current records were filtered at $1-3 \mathrm{kHz}$, digitized with the use of the Digidata 1200 interface $(10 \mathrm{kHz}$; Axon Instruments), and stored on an IBM PC-compatible computer by sampling at $5 \mathrm{kHz}$ for off-line analysis with the use of pClamp6 software (Axon Instruments).

Quantal analysis. Binned EPSC amplitude histograms were fitted with sums of Gaussian curves using a least squares criterion (pStat program in pClamp6). In the majority of experiments, peaks in the amplitude histograms were clearly detectable by eye. The fit was restricted by the number of Gaussian curves and the amplitude range to be fitted. When fitting with multiple-component Gaussian curves, pStat requires entering of estimates (seed values) of the fitting function parameters (the fractional contribution of each component to the area under the curve, the mean of each component corresponding to the peak of the Gaussian distribution, and the SD of the Gaussian distribution) in advance. These optimal parameters were determined by finding the best fit in the observed amplitude histogram. The bin width was chosen to ensure that at least five bins lay between two neighboring peaks. For all distributions, least squares fits were made at least twice with different bin widths, and in no case were significant differences seen. The error function for the least squares fit was minimized with a Simplex method. The value of quantal size $(q)$ was calculated as the mean peak separation using the following equation (Jonas et al., 1993): $q=1 / N \Sigma M_{\mathrm{k}} / k$ ( $N$, total number of peaks; $M_{\mathrm{k}}$, mean amplitude of the $k$ th Gaussian component; and $k$, peak number). The peaks were considered equidistant if the SD of the $M_{\mathrm{k}} / k$ values was $<8 \%$ of their mean $(q)$.

All values in the text give the mean $\pm \mathrm{SD}$. Statistical analysis was made using two-tailed $t$ tests with a significance limit of $p<0.05$.

\section{RESULTS}

\section{Unitary synaptic currents in segmental} parasympathetic reflex pathways

EPSCs were evoked in PGNs by stimulating interneurons located immediately dorsal to the lumbosacral parasympathetic nucleus (Fig. 1). These interneurons are thought to mediate disynaptic activation of PGNs by primary afferents in the segmental parasympathetic reflex pathway (McMahon and Morrison, 1982; Birder and de Groat, 1992; Nadelhaft and Vera, 1995; Araki and de Groat, 1996; de Groat et al., 1996). This notion was examined by recording synaptic responses from the interneurons following field stimulation of primary afferent fibers located at the lateral edge of the dorsal horn (LCP) (Nadelhaft and Booth, 1984; McKenna and Nadelhaft, 1986). In 9 of 11 dorsal interneurons tested, EPSCs could be evoked by stimulating the LCP (Fig. $2 A$ ). These evoked responses were monosynaptic in nature, because the latency was short $(2.2 \pm 0.3 \mathrm{msec} ; n=9)$ and constant during high-frequency stimulation $(20 \mathrm{~Hz})$.

Electrical stimulation of single dorsal interneurons evoked EPSCs in PGNs identified by retrograde labeling (Araki, 1994; Araki and de Groat, 1996). These EPSCs were considered to result from activation of a single presynaptic neuron (i.e., unitary responses), based on the following criteria (Takahashi, 1992; Jonas et al., 1993; Araki and de Groat, 1996): (1) when the stimulating pipette was moved $10-30 \mu \mathrm{m}$ away from the interneuron, stimulus intensities two to four times higher than the original threshold stimulus could not evoke synaptic responses in PGNs; (2) the latency and the rise time distributions of individual EPSCs showed a single sharp peak (data not shown) (Araki and de Groat, 1996); and (3) the mean EPSC peak amplitude showed all-or-none behavior as a function of stimulus intensity (Fig. $2 B, C)$.

These synaptic currents were completely blocked by 6-cyano7-nitroquinoxaline $(5 \mu \mathrm{M})$ and 2-amino-5-phosphonovalerate (50 $\mu \mathrm{M}$ ), indicating that they are glutamatergic EPSCs (Araki and de Groat, 1996).

\section{Developmental synaptic depression}

We examined whether the mean magnitude of unitary EPSCs elicited in PGNs by stimulation of the interneuron might change in the early postnatal period, from 1 to 3 weeks after birth. As shown in Figure 3, the mean peak amplitude of EPSCs recorded at a holding potential of $-60 \mathrm{mV}$ was unchanged during the first (6-8 d) and second (13-15 d) postnatal weeks ( $39.5 \pm 20.7 \mathrm{pA}$; $n=14$; and $37.4 \pm 15.3 \mathrm{pA} ; n=15$, respectively) but was markedly reduced at 3 weeks of age (20-22 d) $(19.3 \pm 8.9 \mathrm{pA} ; n=$ 14; $p<0.01)$. The time course of the EPSCs and other parameters (input capacitance and input resistance) did not differ at these three periods.

Maturation of the descending inputs from the brain has been suggested to play an important role in the suppression of segmental reflexes during the postnatal period (de Groat et al., 1975, 1993). To examine this possibility, the spinal cord was transected at the $\mathrm{T}_{10}$ segment on postnatal day 14 . This procedure eliminated segmental synaptic depression expected to occur at 3 weeks of age (38.2 $\pm 21.6 \mathrm{pA} ; n=15)$ (Fig. 3, open circle). Thus, the developmental synaptic depression appears to require the formation of the descending inputs.

\section{Reduction in the transmitter release}

The reduced synaptic efficacy could be a result of a reduction in the amount of the transmitter released from presynaptic terminals 
or a reduction in the postsynaptic sensitivity to the transmitter. We conducted a quantal analysis to examine these possible mechanisms (Redman, 1990). In 36 cells that had a sufficient sample size (>300 responses), the peak amplitude histograms of the unitary EPSCs could be well fitted by the sum of several Gaussian functions with equidistant peaks, indicating quantal release of the transmitter at the interneuronal-PGN synapse (Fig. 4). The mean quantal size (Fig. $5 A$ ) measured at a holding potential of $-60 \mathrm{mV}$ was $10.3 \pm 0.3 \mathrm{pA}(n=14)$ at $1-2$ weeks of age. A similar value was obtained in preparations from 3 -week-old rats with an intact $(10.1 \pm 0.5 \mathrm{pA} ; n=11)$ or a transected $(10.4 \pm 0.5 \mathrm{pA} ; n=11)$ spinal cord. The quantal conductance change, calculated assuming a reversal potential of $0 \mathrm{mV}$, was $\sim 170 \mathrm{pS}$ during these postnatal periods. These results imply that the postsynaptic sensitivity to the transmitter remained unchanged during development.

In 10 cells, the release probability was reduced by lowering $\mathrm{Ca}^{2+}$ and increasing $\mathrm{Mg}^{2+}$ concentrations in the external solution. Under this condition, the frequency of failures increased, and the mean peak amplitude of EPSCs was decreased without any change in quantal size (Fig. $4 A, b$ ). Thus, the constancy of the quantal size also suggests that the reduction of evoked synaptic responses results from a reduced quantal content of the transmitter release.
When the mean quantal content was calculated by dividing the mean amplitude of unitary EPSCs by the quantal size in each cell (Fig. $5 B)$, it was significantly reduced at 3 weeks of age $(1.8 \pm 0.9$; $n=11)$ compared with 1 - or 2 -week-old rats $(3.3 \pm 1.6 ; n=14$; $p<0.03)$. However, the developmental decrease in transmitter release normally seen at 3 weeks of age was eliminated after chronic spinal cord transection $(3.8 \pm 2.1 ; n=11 ; p<0.01)$. Essentially, the same result was obtained when the mean quantal content was estimated from the number of failures (Fig. $5 C$ ). During the first 3 postnatal weeks, this quantal content was reduced from $2.5 \pm 0.4(n=14 ; 1-2$ weeks of age $)$ to $1.5 \pm 0.3$ ( $n=11 ; p<0.001 ; 3$ weeks of age) in intact animals but not in spinalized rats $(2.4 \pm 0.6 ; n=11)$. Thus, this developmental synaptic depression is most likely attributed to a reduction in the number of quanta released from presynaptic terminals.

\section{DISCUSSION}

The interneurons stimulated in our experiments are located in the region of the intermediate gray matter that receives a prominent input from the somatovisceral primary afferent pathway (Nadelhaft and Booth, 1984; McKenna and Nadelhaft, 1986). The primary afferents enter Lissauer's tract and then send collaterals ventrally along the lateral edge of the dorsal horn (LCP) into the dorsal region of the SPN. Somatovisceral stimulation can activate
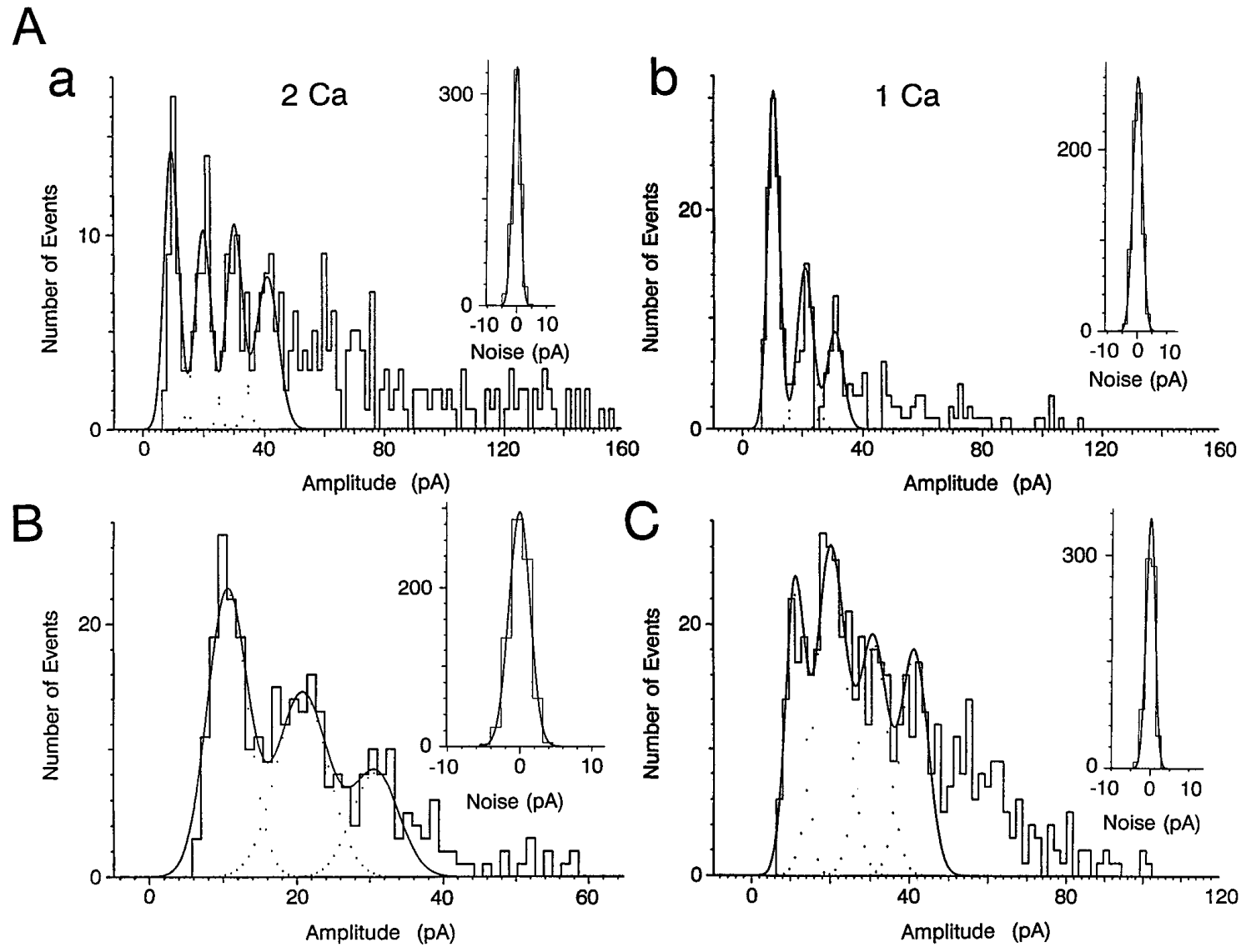

Figure 4. Quantal analysis indicated a presynaptic mechanism for the developmental synaptic depression. Peak amplitude distributions of unitary EPSCs recorded from PGNs at a holding potential of $-60 \mathrm{mV}$. A, From an 8-d-old rat. Bin width was $1.6 \mathrm{pA}$. $a$, In the standard external solution (2 mm $\mathrm{Ca}^{2+}$ and $1 \mathrm{mM} \mathrm{Mg}^{2+}$ ), 23 failures in 339 trials; $b$, in an external solution containing $1 \mathrm{mM} \mathrm{Ca}^{2+}$ and $5 \mathrm{mM} \mathrm{Mg}^{2+}$ to reduce the release probability, 124 failures in 363 trials. $B$, From a 20-d-old rat. Bin width was 1.2 pA, 48 failures in 363 trials. $C$, From a 21-d-old rat spinalized at $\mathrm{T}_{10}$ level 2 weeks after birth. Bin width was $1.6 \mathrm{pA}, 27$ failures in 581 trials. Insets, Frequency distributions of background noise. The smooth lines superimposed on the histograms represent the sum of Gaussian distributions that best fit the data. In all recordings, the SD of the noise $(1.5 \pm 0.3 \mathrm{pA} ; n=31)$ was less than that of any Gaussian curve fitted to the EPSC amplitude histogram. The failures of response are not shown. 
A

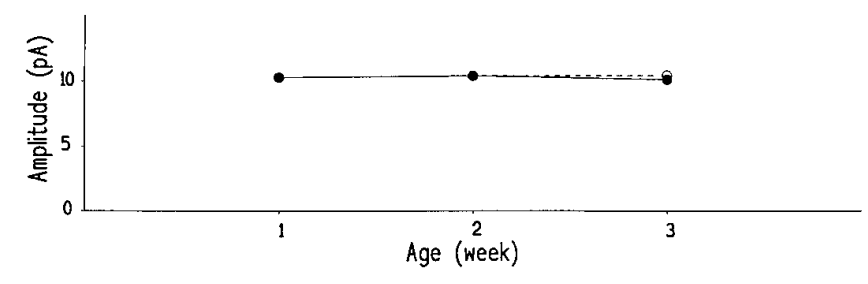

B

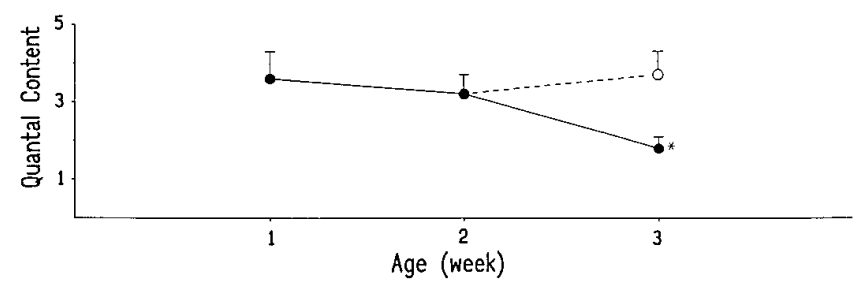

C

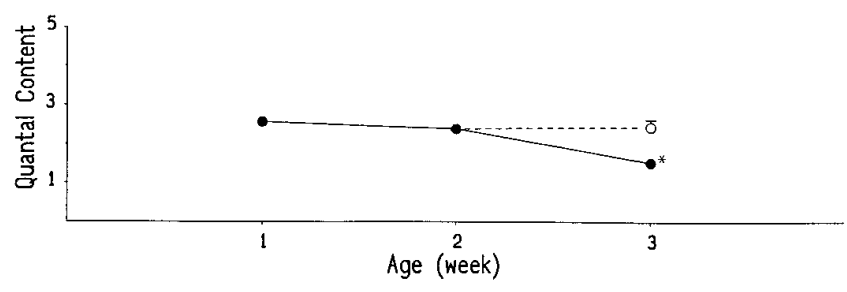

Figure 5. Developmental changes of quantal size (quantal amplitude) and quantal content. $A$, Mean quantal amplitudes measured at a holding potential of $-60 \mathrm{mV}$. $B$, Mean quantal contents calculated by dividing the mean amplitude of unitary EPSCs by the quantal size in each cell. $C$, Mean quantal contents calculated from the following equation, based on Poisson's law: $m=\log _{\mathrm{e}}\left(N / n_{0}\right)$ ( $m$, quantal content; $N$, total number of trials; and $n_{0}$, number of failures of response). Mean values obtained from PGNs in intact $(\bullet)$ or spinalized $(\bigcirc)$ rats are plotted against ages. Each mean value was measured from 7 or 11 cells in which $>300$ responses were evoked. Each point represents the mean \pm SEM (vertical bar). SEMs $<0.15$ are omitted. The asterisk represents significant difference from the values observed in other preparations $(p<0.03)$.

the interneurons in this region (McMahon and Morrison, 1982; Birder and de Groat, 1992), which in turn form excitatory synapses on PGNs (Nadelhaft and Vera, 1995; Araki and de Groat, 1996). Thus, the dorsal interneurons could function as relay neurons in a disynaptic excitatory pathway from primary afferents to the PGN (Araki and de Groat, 1996; de Groat et al., 1996). Consistent with this notion, monosynaptic EPSCs were evoked in the interneurons by focal electrical stimulation of the LCP.

We did not explore the detailed time course of changes in synaptic transmission after spinal transection. At synapses formed by Ia sensory fibers on motoneurons, enhancement of synaptic efficacy occurs in two forms after spinal transection (Mendell, 1984). The acute form lasts only a few days, whereas the chronic form develops more slowly. The chronic changes have been suggested to be caused by collateral sprouting or activation of the already existing but nonfunctional synapses, in contrast with some nonspecific process (e.g., humoral factors) responsible presumably for the acute change (Nelson et al., 1979; Cope et al., 1980; Mendell, 1984). These chronic processes appear to require partial deafferentation of motoneurons by elimination of descending and/or segmental inputs. Spinal transection at the thoracic level cannot particularly disrupt the segmental inputs to

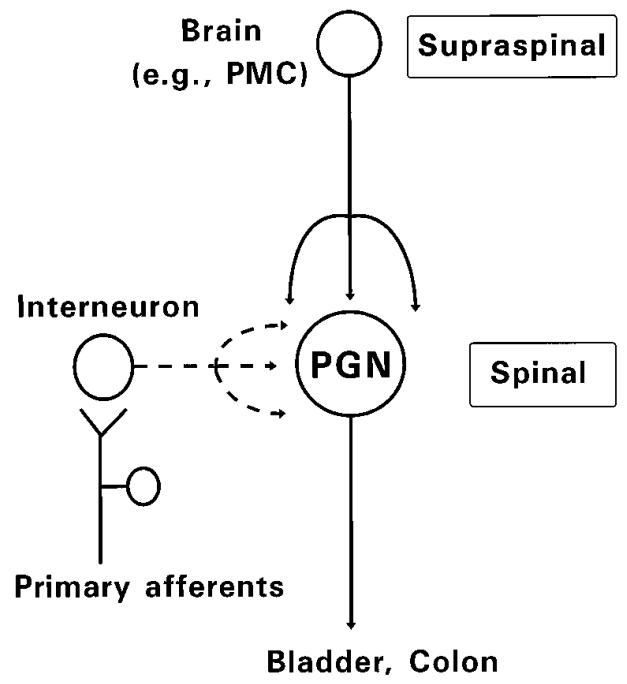

Figure 6. Diagram indicating two sources of excitatory inputs to PGNs during development. In neonates (broken lines), interneurons synaptically activated by primary afferent inputs mediate segmental somatovisceral reflexes. In adults (solid lines), descending projections from higher center in the brain (e.g., $P M C$ ) control PGN activity. The interneuronal inputs and the bulbospinal inputs could compete for synaptic sites on PGNs during postnatal development.

PGNs in the lumbosacral cord. Thus, it seems likely that elimination of the descending inputs is the primary cause for the changes in synaptic transmission to PGNs observed after transection in our study.

The major finding in the present study was abrupt depression of excitatory transmission at interneuronal-PGN synapses in segmental parasympathetic reflex pathways during the third postnatal week. This coincides with the time when the spinobulbospinal reflex via the pontine micturition center (PMC) replaces the segmental spinal reflex as the principal mechanism for micturition (de Groat, 1975; Kruse and de Groat, 1990; de Groat et al., 1993). In addition, chronic spinal transection prevented the developmental synaptic depression. This result is consistent with the observations that primitive excretory reflex patterns persist or reappear in chronic spinalized animals (de Groat et al., 1975, 1993; Kruse and de Groat, 1990). Thus, this synaptic depression could be a mechanism that underlies the suppression of primitive segmental reflexes during development.

Synaptic depression in the segmental pathway was attributable to a reduction in the amount of transmitter release. At present, it is not clear how transmitter release (quantal content) is reduced. Although no morphological data are currently available, a progressive elimination of synapses formed on a PGN by an interneuron may occur, as noted for the competitive refinement of synaptic connections during early postnatal development (Purves and Lichtman, 1985; Goodman and Shatz, 1993). At the neuromuscular junction a reduction of quantal content is reported to occur before synapse elimination (axonal withdrawal) (Colman et al., 1997). It has been suggested recently that some synapses can be converted to nonfunctional at normal resting potentials by expressing only NMDA receptors but not AMPA receptors in postsynaptic neuron (Liao et al., 1995). This "silent synapse" mechanism may be implicated in developmental synaptic refinements, as suggested in the hippocampus and the barrel cortex (Durand et al., 1996; Isaac et al. 1997). However, it is still premature to elaborate this mechanism further. 
The descending projections from the brain (e.g., PMC), which mature during postnatal development (Gilbert and Stelzner, 1979), very likely compete with segmental interneuronal inputs for synaptic space on PGNs (Fig. 6). Indeed, the elimination of the bulbospinal inputs by chronic spinal transection prevented this developmental synaptic depression. Thus, we conclude that developmental modification of excitatory synapses on PGNs is attributable to competition between their segmental and suprasegmental inputs, which results in the reorganization of parasympathetic excretory reflex pathways. If so, competitive synaptic refinements could serve as a switching mechanism to replace neural reflex pathways during development, in addition to making more subtle adjustments in homogeneous overlapping synaptic connections that have been reported in other systems (Purves and Lichtman, 1985; Goodman and Shatz, 1993).

It is known that the pattern of electrical activity is a key factor in synaptic competition in the developing nervous system (Ridge and Betz, 1984; Fregnac et al., 1988; Constantine-Paton et al., 1990; Dan and Poo, 1992; Goodman and Shatz, 1993; BaliceGorden and Lichtman, 1994) as well as in functional plasticity at mature synapses (Madison et al., 1991; Linden, 1994). Although mechanisms for the developmental synaptic plasticity identified in this study remain to be explored, this slice preparation, which allows the study of unitary synaptic connections between identified pairs of neurons, will be useful in elucidating the cellular mechanisms underlying the refinement of synaptic connections in the developing CNS.

\section{REFERENCES}

Anderson CR, Edwards SL (1994) Intraperitoneal injections of fluorogold reliably labels all sympathetic preganglionic neurons in the rat. J Neurosci Methods 53:137-141.

Araki I (1994) Inhibitory postsynaptic currents and the effects of GABA on visually identified sacral parasympathetic preganglionic neurons in neonatal rats. J Neurophysiol 72:2903-2910.

Araki I, de Groat WC (1996) Unitary excitatory synaptic currents in preganglionic neurons mediated by two distinct groups of interneurons in neonatal rat sacral parasympathetic nucleus. J Neurophysiol 76:215-226.

Balice-Gorden RJ, Lichtman JW (1994) Long-term synapse loss induced by focal blockade of postsynaptic receptors. Nature 372:519-524.

Beach FA (1966) Ontogeny of "coitus-related" reflexes in the female guinea pig. Proc Natl Acad Sci USA 56:526-533.

Birder LA, de Groat WC (1992) Induction of c-fos gene expression of spinal neurons in the rat by nociceptive and non-nociceptive stimulation of the lower urinary tract. Am J Physiol 265:R326-R333.

Colman H, Nabekura J, Lichtman JW (1997) Alterations in synaptic strength preceding axon withdrawal. Science 275:356-361.

Constantine-Paton M, Cline HT, Debski E (1990) Patterned activity, synaptic convergence, and the NMDA receptor in developing visual pathways. Annu Rev Neurosci 13:129-154.

Cope TS, Nelson SG, Mendell LM (1980) Factors outside neuraxis mediate "acute" increase in EPSP amplitude caudal to spinal cord transection. J Neurophysiol 44:174-183.

Dan Y, Poo M (1992) Hebbian depression of isolated neuromuscular synapses in vitro. Science 256:1570-1573.

de Groat WC (1975) Nervous control of the urinary bladder of the cat. Brain Res 87:201-211.

de Groat WC, Douglas JW, Glass J, Simonds W, Weimer B, Werner P (1975) Changes in somato-vesical reflexes during postnatal development in the kitten. Brain Res 94:150-154.

de Groat WC, Booth AM, Yoshimura N (1993) Neurophysiology of micturition and its modification in animal models of human disease. In: The autonomic nervous system, Vol 3 (Maggi CA, ed), pp 227-290. London: Harwood.

de Groat WC, Vizzard MA, Araki I, Roppolo J (1996) Spinal interneurons and preganglionic neurons in sacral autonomic pathways. Prog Brain Res 107:97-111.
Durand GM, Kovalchuk Y, Konnerth A (1996) Long-term potentiation and functional synapse induction in developing hippocampus. Nature 381:71-75.

Edwards FA, Konnerth A, Sakmann B, Takahashi T (1989) A thin slice preparation for patch clamp recordings from neurons of the mammalian central nervous system. Pflügers Arch 414:600-612.

Fregnac Y, Shulz D, Thorpe S, Bienenstock E (1988) A cellular analogue of visual cortical plasticity. Nature 333:367-370.

Fukuda H, Fukai K, Yamane M, Okada H (1981) Pontine reticular unit responses to pelvic nerve and colonic mechanical stimulation in the dog. Brain Res 207:59-71.

Gilbert M, Stelzner DJ (1979) The development of descending and dorsal root connections in the lumbosacral spinal cord of the postnatal rat. J Comp Neurol 184:821-838.

Goodman CS, Shatz CJ (1993) Developmental mechanisms that generate precise patterns of neuronal connectivity. Cell 72/Neuron 10[Suppl]:77-98.

Hubel DH, Wiesel TN (1965) Binocular interaction in striate cortex of kittens reared with artificial squint. J Neurophysiol 28:1041-1059.

Isaac JTR, Crair MC, Nicoll RA, Malenka RC (1997) Silent synapses during development of thalamocortical inputs. Neuron 18:269-280.

Jonas P, Major G, Sakmann B (1993) Quantal components of unitary EPSCs at the mossy fibre synapse on CA3 pyramidal cells of rat hippocampus. J Physiol (Lond) 472:615-663.

Kruse MN, de Groat WC (1990) Micturition reflexes in decerebrate and spinalized neonatal rats. Am J Physiol 258:R1508-R1511.

Liao D, Hessler NA, Malinow R (1995) Activation of postsynaptically silent synapses during pairing-induced LTP in CA1 region of hippocampal slice. Nature 375:400-404.

Linden DJ (1994) Long-term synaptic depression in the mammalian brain. Neuron 12:457-472.

Madison DV, Malenka RC, Nicoll RA (1991) Mechanisms underlying long-term potentiation of synaptic transmission. Annu Rev Neurosci 14:379-397.

Manabe T, Araki I, Takahashi T, Kuno M (1991) Membrane currents recorded from sexually dimorphic motoneurones of the bulbocavernosus muscle in neonatal rats. J Physiol (Lond) 40:419-435.

McKenna KE, Nadelhaft I (1986) The organization of the pudendal nerve in the male and female rat. J Comp Neurol 248:532-549.

McMahon SB, Morrison JFB (1982) Two groups of spinal interneurones that respond to stimulation of the abdominal viscera of the cat. J Physiol (Lond) 322:21-34.

Mendell LM (1984) Modifiability of spinal synapses. Physiol Rev 64:260-324.

Nadelhaft I, Booth AM (1984) The location and morphology of preganglionic neurones and the distribution of visceral afferents from the rat pelvic nerve: a horseradish peroxidase study. J Comp Neurol 226:238-245.

Nadelhaft I, Vera PL (1995) Central nervous system neurons infected by pseudorabies virus injected into the rat urinary bladder following unilateral transection of the pelvic nerve. J Comp Neurol 359:443-456.

Nelson SG, Mendell LM (1979) Enhancement in Ia-motoneuron synaptic transmission caudal to chronic spinal transection. J Neurophysiol 42:642-654.

Purves D, Lichtman JW (1985) Principles of neural development. Sunderland, MA: Sinauer.

Redfern PA (1970) Neuromuscular transmission in new-born rats. J Physiol (Lond) 209:701-709.

Redman S (1990) Quantal analysis of synaptic potentials in neurons of the central nervous system. Physiol Rev 70:165-198.

Reiter HO, Stryker MP (1988) Neural plasticity without post-synaptic action potentials: Less-active inputs become dominant when kitten visual cortical cells are pharmacologically inhibited. Proc Natl Acad Sci USA 85:3623-3627.

Ridge RMAP, Betz WJ (1984) The effect of selective, chronic stimulation on motor unit size in developing rat muscle. J Neurosci 4:2614-2620.

Takahashi T (1992) The minimal inhibitory synaptic currents evoked in neonatal rat motoneurones. J Physiol (Lond) 450:593-611.

Thor KB, Blais DP, de Groat WC (1989) Behavioral analysis of the postnatal development of micturition in kittens. Brain Res 46:137-144. 